\title{
IgA immune complex deposits, what can lie behind them? Comments on a case of IgA dominant glomerulonephritis associated to staphylococcus infection.
}

\author{
Fernanda Carvalho \\ Nephropathologist in Nefrolab, Paço de Arcos
}

In this journal Nicole Pestana et al. describe a case of "IgA dominant glomerulonephritis associated to staphylococcus infection". They draw attention to clinical aspects, difficulty of diagnosis and differential diagnosis, and treatment.

In the last two decades we have witnessed the decline of streptococcal infections and the near disappearance of acute streptococcal glomerulonephritis. The more recent published literature focuses on glomerulonephritis associated staphylococcus infection ${ }^{1}$. These conditions are frequently characterized by the presence of glomerular dominant IgA deposits and are known worldwide as IgA dominant staphylococcal glomerulonephritis. Glomerular IgA deposit is the central feature in these circumstances, crucial for the diagnosis but occurring in many other conditions, requiring different approaches.

When immunofluorescence detects a dominant IgA immune complex deposit in the glomeruli of a renal biopsy, a large number of conditions may be behind this finding, ranging from IgA nephropathy (Berger), IgA vasculitis (Henoch-SchÖnlein purpura), immune diseases, minimal change disease with IgA, membranous and IgA glomerulonephritis, secondary IgA diseases to glomerulonephritis related infectious diseases with IgA dominant deposits.

In the nineties, glomerulonephritis related staphylococcal infections were only described as secondary to endocarditis, visceral abscesses and ventricular shunts. Nowadays, the published literature shows us that there is an association between staphylococcus infection and glomerulonephritis, not only related to those conditions. The majority of these infections is persistent and caused by methicillin-resistant strains of Staphylococcus aureus. Haas, et al. ${ }^{2}$ and Nasr, et al ${ }^{3}$ report a biopsy incidence for that condition of $0.2 \%$ (13 of 6334) and $0.9 \%$ (93 of 10,080 ) respectively. The patients were all adults, over 50 years of age and with large panoply of site infections, including osteomyelitis, septic arthritis, pneumonia, infected leg ulcer, rectal abscess and other deep-seated abscesses. Blood cultures may be negative, because patients are often, arbitrarily, treated with antibiotics, contrary to what happens with staphylococcus endocarditis infection. Most patients are elderly with comorbidities such as diabetes, malignancy, congestive heart failure, bypass arterial graft or stent. Patients frequently present acute renal failure, microscopic hematuria and proteinuria, which may be severe. Purpuric skin rash may appear, raising difficulties for a differential diagnosis with IgA vasculitis. Test for cryoglobulins is rarely positive; C3 is low in a small percentage of patients, only in the series of $\mathrm{Nasr}^{3}$ did $72 \%$ of the patients have hypocomplementemia.

Pathologic findings are multiple. Enlarged glomeruli with mesangioproliferative or endocapillary pattern of proliferation and polymorphonuclear leukocytes similar to poststreptococcal infection may be observed by light microscopy. More rarely a membranoproliferative pattern may be present. Crescents may occur as necrotizing lesions. In these cases, differential diagnosis with PSH (which is a rare condition in the elderly) and ANCA associated vasculitis may become difficult.

Immunofluorescence reveals the IgA deposit in glomeruli. The deposits may be diffuse or focal, global or segmental. They may appear as bright or trace, predominantly in mesangium or along the capillary loops. C3 is, in general, abundant, coarsely granular and bright. As in IgA nephropathy, lambda light chain is stronger than Kappa light chain.

Electron microscopy reveals the deposits in mesangium and in some cases also localized in the subendothelial or subepithelial spaces. Some cases may have "humps", but they are not specific to this condition 4 .

A rare but growing condition, the main problem of IgA dominant staphylococcal glomerulonephritis is to differentiate this condition from the vast series of IgA diseases, taking into account the difference in treatment. This is a laborious diagnosis, as emphasized by Nicole Pestana et al. Morphology may help but in some cases such as PSH and others it is still very difficult to rely solely on morphology, because of the great similarity of the morphologic findings of the various conditions. Diagnosis will always be more secure if supported by well-detailed clinical-laboratory data with utmost attention to bacteriology and serology. Should an adult patient have symptoms of IgA vasculitis and a renal biopsy showing IgA-dominant glomerulonephritis, a staphylococcal infection could be the cause.

\section{Disclosure of potential conflicts of interest: none declared.}

\section{References}

1. Charles Jennette, Vivete D'Agati, Jean Olson, Fred Silva. Heptinstall's Pathology of the Kidney. 7th Edition. Lippincott, 2014: 676-798

2. Haas M, Racusen L, Bagnasco S. IgA-dominant postinfectious glomerulonephritis: a report of 13 cases with common ultrastructural features. Hum Pathol 2008; 39:1309-1316.

3. Nasr SH, Radhakrishnan J, D'Agati VD. Bacterial infection-related glomerulonephritis in adults. Kidney Int 2013; 83(5):792-803. doi: 10. 1038/ki.2012.407.

4. Nasr S, Fidler M, Valeri A, et al. Postinfectious glomerulonephritis in the elderly. J Am Soc Nephrol 2011; 22:187-195.

\section{Correspondence to:}

Fernanda Carvalho, MD

Nefrolab - Paço de Arcos;

Rua Gago Coutinho e Sacadura Cabral, no 136/138 2955-190 Pinhal Novo.

E-mail: fanecarvalho@gmail.com 